\title{
Progressive dyspnea associated with a crazy-paving appearance on a chest computed tomography scan
}

\author{
Nimrod Maimon MD ${ }^{1}$, Narinder Paul MD FRCR(UK) FRCPC ${ }^{2}$, Gregory P Downey MD FRCPC FCCP ${ }^{3}$
}

N Maimon, N Paul, GP Downey. Progressive dyspnea associated with a crazy-paving appearance on a chest computed tomography scan. Can Respir J 2006;13(5):269-271.

\begin{abstract}
A 'crazy-paving' appearance of the lungs on computed tomography scanning of the chest was first described nearly 20 years ago in patients with pulmonary alveolar proteinosis, and was thought to be characteristic of this condition. However, this pattern has subsequently been reported in a variety of pulmonary diseases and is now considered to be nonspecific. The present report describes a case of a 74-year-old man in whom congestive heart failure presented with a crazy-paving appearance of the lungs on a chest computed tomography scan. This uncommon association illustrates the importance of the correlation of clinical and radiographic information.
\end{abstract}

\section{Dyspnée progressive associée à un aspect en verre dépoli à la scintigraphie thoracique}

\begin{abstract}
Un aspect en verre dépoli à la scintigraphie pulmonaire a été décrit pour la première fois il y a près de 20 ans chez des patients qui souffraient de protéinose alvéolaire pulmonaire et on croyait qu'il était caractéristique de cette maladie. Or, ce motif a par la suite été signalé en lien avec diverses maladies pulmonaires et est désormais considéré non spécifique. Le présent rapport décrit le cas d'un homme de 74 ans atteint d'insuffisance cardiaque congestive chez qui on a noté à la scintigraphie pulmonaire un aspect en verre dépoli. Ce signe rare illustre l'importance d'établir une corrélation entre les renseignements cliniques et radiographiques.
\end{abstract}

Key Words: Computed tomography; Congestive heart failure; Crazy-paving appearance; Pulmonary alveolar proteinosis

A 'crazy-paving' appearance of the lungs on a high-resolution computed tomography (CT) scan of the chest, defined as scattered or diffuse ground-glass attenuation superimposed on a network of interlobular septal thickening and intralobular lines, was first described in association with pulmonary alveolar proteinosis nearly 20 years ago $(1,2)$. Initially, this radiographic pattern was thought to be specific for alveolar proteinosis, but has subsequently been reported in a variety of interstitial and airspace pulmonary disorders $(3,4)$. In the present report, we describe a man who presented with progressive exertional dyspnea in association with a high-resolution chest CT scan that demonstrated a typical crazy-paving appearance. Subsequent investigations and his clinical course demonstrated the cause for the abnormal radiographic changes to be pulmonary edema secondary to congestive heart failure. The association of cardiogenic pulmonary edema, a common condition, with a crazy-paving appearance on a CT scan of the lungs has been reported previously in only three cases, but it is important for clinicians and radiologists to recognize.

\section{CASE PRESENTATION}

A 74-year-old man presented with a nine-week history of progressive exertional dyspnea and fatigue during which time his exercise tolerance declined from almost unlimited to less than one flight of stairs. There was no history of fever, cough, weight loss, chest pain or peripheral edema. His past medical history included high-grade prostate cancer for which he was treated with local radiation and hormonal therapy that ended two weeks before hospital admission. Medical records indicated that there was local control of the cancer without evidence of local or distant metastasis. He also had a history of coronary artery disease in association with the cardiac risk factors of arterial hypertension and hypercholesterolemia. An echocardiogram one year earlier demonstrated mild to moderate left ventricular dysfunction. He had a remote history of deep venous thrombosis in his left leg. The patient also had a clinical history of asbestos-related pleural disease that was attributed to asbestos exposure while working in the British shipping industry 50 years previously. He was followed regularly by his family physician for these problems. His current medications included acetylsalicylic acid, atorvastatin, nifedipine and fluoxetine. He was an ex-smoker (with 50-pack year exposure) who had quit smoking 10 years ago. He drank one glass of wine per day and had no known allergies, no pets and no history of recent travel.

On physical examination he appeared well and in no respiratory distress. The following parameters were recorded: blood pressure of $135 / 70 \mathrm{mmHg}$, heart rate of 76 beats/min, respiratory rate of 24 breaths $/ \mathrm{min}$ and a body temperature of $37.2^{\circ} \mathrm{C}$. His arterial oxygen saturation on room air was $86 \%$. His jugular venous pressure was not elevated. Cardiac examination revealed a normal S1 and diminished S2, with no murmurs or extra sounds. Auscultation of the chest revealed inspiratory crackles bilaterally. His abdominal examination was unremarkable without organomegaly. There was no peripheral cyanosis, clubbing or edema. Laboratory findings revealed a normal complete blood count and differential blood count. Serum

\footnotetext{
${ }^{1}$ Division of Respirology, Department of Medicine, University of Toronto; ${ }^{2}$ Department of Radiology; ${ }^{3}$ Department of Medicine, Toronto General Hospital, University Health Network, Toronto, Ontario

Correspondence: Dr Gregory P Downey, Room 6264 - Medical Sciences Building, University of Toronto, 1 King's College Circle, Toronto, Ontario M5S 1A8. Telephone 416-340-4591, fax 416-340-3109, e-mail gregory.downey@utoronto.ca
} 


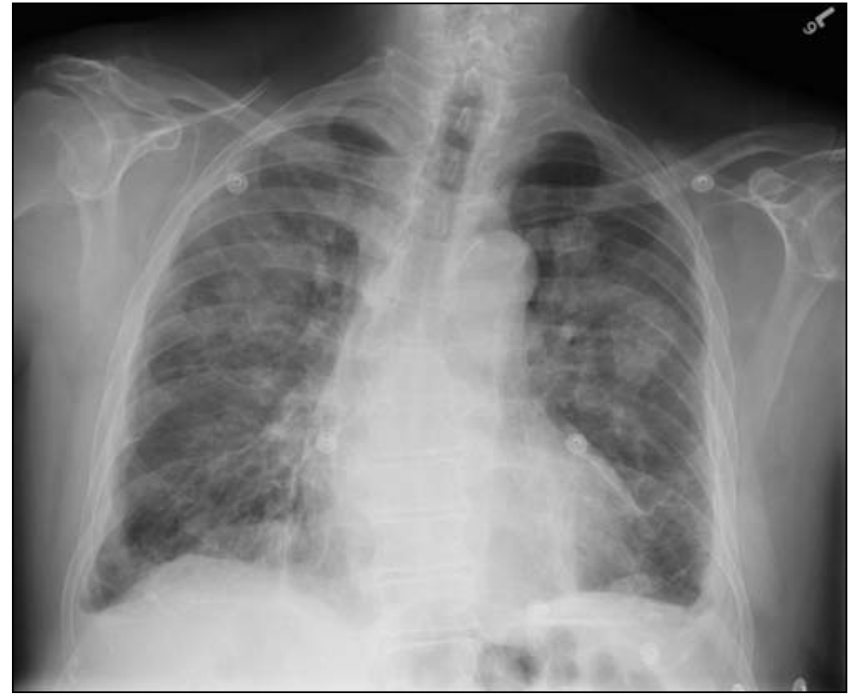

Figure 1) Chest radiograph illustrating diffuse bilateral airspace opacities affecting both lung fields, with evidence of pleural thickening consistent with previous asbestos exposure

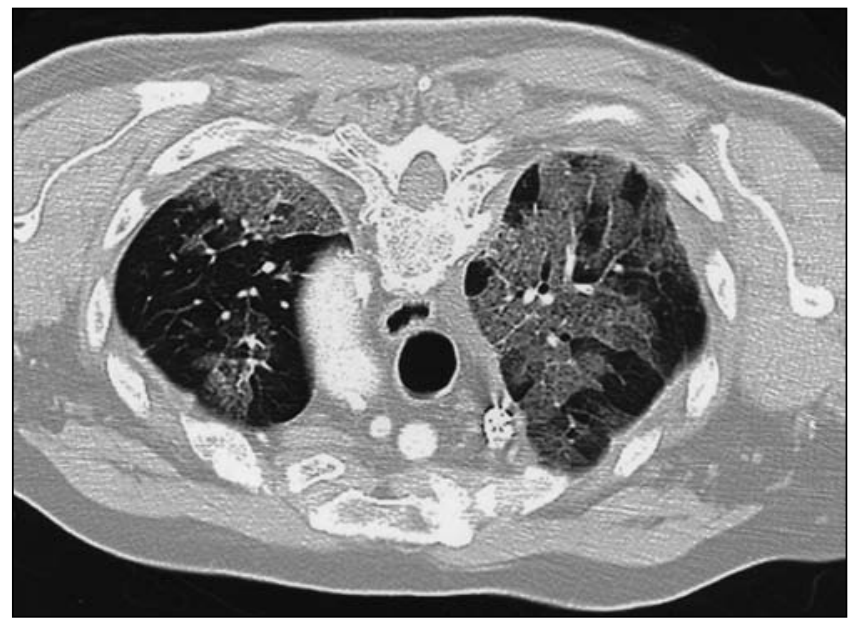

Figure 2) Chest computed tomography scan showing ground-glass opacities superimposed on interlobular septal thickening consistent with a crazy-paving pattern

electrolytes, liver and renal function tests were all normal. The level of troponin I was $0.6 \mathrm{ng} / \mathrm{L}$, within the normal range. Arterial blood gas analysis on room air revealed a $\mathrm{pH}$ of 7.35, a partial pressure of carbon dioxide of $36 \mathrm{mmHg}$, a partial pressure of oxygen of $55 \mathrm{mmHg}$ and a bicarbonate concentration of $24 \mathrm{mmol} / \mathrm{L}$.

A chest radiograph demonstrated diffuse airspace opacities affecting both lungs, on a background of bilateral pleural thickening and calcifications consistent with previous asbestos exposure (Figure 1). A CT scan of the chest was obtained using a pulmonary embolism protocol to rule out pulmonary embolism (Figures 2 and 3). The scan revealed calcification of all three coronary arteries. No mediastinal lymphadenopathy was noted. There were small linear opacities in the lung, and bilateral pleural thickening and calcification on the lateral and diaphragmatic surfaces. A small pleural effusion was noted. In both upper lobes, there were diffuse, multifocal, patchy, ground-glass opacities with superimposed reticulations resulting in a crazy-paving appearance. The CT scan was compared

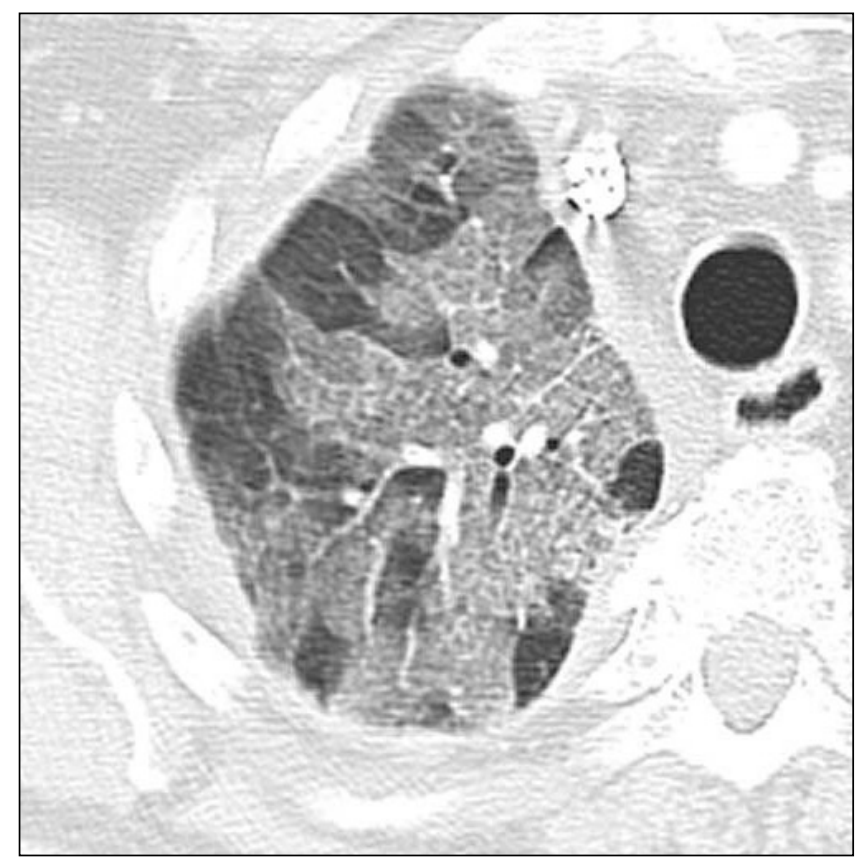

Figure 3) Chest computed tomography scan showing ground-glass opacities superimposed on interlobular septal thickening consistent with a crazy-paving pattern

with a previous study performed six years earlier that showed only minor interstitial linear opacities, and pleural thickening and calcification consistent with his history of asbestos exposure. No signs of a crazy-paving appearance were apparent on the earlier CT scan.

The patient underwent bronchoscopy with bronchoalveolar lavage from the right upper lobe and transbronchial biopsies from the right middle lobe. The lavage fluid was sent for routine cytology, cell count and differential count, Gram stain and bacterial culture, acid-fast stain and mycobacterial culture, and fungal stain and culture; all tests were negative. The transbronchial biopsies were examined microscopically after hematoxylin and eosin, Gram, trichrome and silver staining, and demonstrated no pathological changes. A two-dimensional echocardiogram demonstrated severe akinesia of the anteroseptal area of the left ventricle, with an ejection fraction between $20 \%$ and $39 \%$. He was treated with intravenous diuretic therapy and improved rapidly.

Subsequent to his discharge, a repeat CT scan of the chest was obtained with $5 \mathrm{~mm}$ reconstructions following multislice acquisition, without contrast media administration. This highresolution study, conducted using $1.3 \mathrm{~mm}$ axial slices at $10 \mathrm{~mm}$ intervals, demonstrated pleural thickening and plaques consistent with prior asbestos exposure, but complete resolution of the crazy-paving appearance (Figure 4).

The combination of a negative laboratory analysis for infection, the echocardiographic demonstration of left ventricular dysfunction, and his clinical course with rapid clinical and radiographic improvement after diuretic therapy led to the conclusion that the cause for the abnormal parenchymal changes on the CT scan of the cheat was pulmonary congestion secondary to heart failure. He was referred to a cardiologist and underwent coronary angiography that demonstrated severe three-vessel coronary artery disease. Coronary artery bypass grafting was recommended. 


\section{DISCUSSION}

The crazy-paving appearance on a high-resolution CT scan of the lungs was first described in patients with pulmonary alveolar proteinosis $(1,2)$. When initially described nearly 20 years ago, this radiographic pattern was thought to be specific for pulmonary alveolar proteinosis. However, this appearance has subsequently been reported in other pulmonary diseases $(3,4)$. A recent study by Johkoh et al (3) retrospectively catalogued the spectrum of diseases associated with the crazy-paving appearance on chest CT scanning. They correlated high-resolution CT images with pathological findings from 20 patients with a crazy-paving appearance who underwent lung biopsy. Histological examination revealed that three different types of processes were associated with a crazy-paving appearance: type 1 was an alveolar filling process, type 2 was an interstitial fibrotic process and type 3 was a combination of both processes.

Based on this study and other information in the literature, it is possible to classify the main pulmonary insults that result in a crazy-paving appearance on an etiological basis into infectious, neoplastic, inhalational, toxic, sanguineous and idiopathic disorders. Specific infectious disorders that are associated with a crazypaving pattern include Pneumocystis jiroveci (carinii) pneumonia, Mycobacterium tuberculosis and Mycoplasma pneumoniae (3). Recently, coronavirus-associated severe acute respiratory syndrome was reported to be associated with a crazy-paving appearance (5). Mucinous bronchioloalveolar carcinoma is the only neoplastic disease reported with this pattern. Talcosis, asbestosis and exogenous lipoid pneumonia are inhalational insults that are associated with a crazy-paving appearance $(3,6,7)$. In this regard, it is noteworthy that the patient described in the present report had a history of asbestos exposure with characteristic calcified pleural plaques. While there was a suggestion on a previous CT scan of interstitial changes, these were not observed on subsequent scans, making asbestos-related pulmonary fibrosis (asbestosis) unlikely. However, it is possible that the patient had a minor degree of asbestosis that was not apparent on the most recent CT scan and that this, in combination with left ventricular failure, contributed to the crazy-paving appearance.

Toxic causes include drug-induced pulmonary toxicity and radiation pneumonitis. The idiopathic group includes pulmonary alveolar proteinosis, sarcoidosis, nonspecific interstitial pneumonitis, usual interstitial pneumonitis, acute interstitial pneumonitis and cryptogenic organizing pneumonia $(3,6-8)$. Pulmonary hemorrhage syndromes and the acute

\section{REFERENCES}

1. Godwin JD, Muller NL, Takasugi JE. Pulmonary alveolar proteinosis: CT findings. Radiology 1988;169:609-13.

2. Murch CR, Carr DH. Computed tomography appearances of pulmonary alveolar proteinosis. Clin Radiol 1989;40:240-3.

3. Johkoh T, Itoh H, Muller NL, et al. Crazy-paving appearance at thin-section CT: Spectrum of disease and pathologic findings. Radiology 1999;211:155-60.

4. Murayama S, Murakami J, Yabuuchi H, Soeda H, Masuda K. "Crazy paving appearance" on high resolution CT in various diseases. J Comput Assist Tomogr 1999;23:749-52.

5. Chan MS, Chan IY, Fung KH, Poon E, Yam LY, Lau KY. High-resolution CT findings in patients with severe acute respiratory syndrome: A pattern-based approach. AJR Am J Roentgenol 2004;182:49-56.

6. Tan RT, Kuzo RS. High-resolution CT findings of mucinous

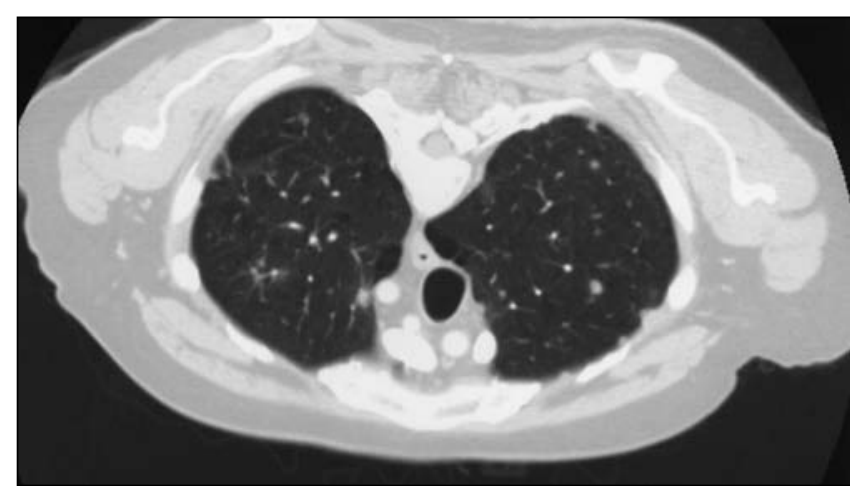

Figure 4) Repeat chest computed tomography scan after diuretic therapy that demonstrates complete resolution of airspace and ground-glass opacities

respiratory distress syndrome have been associated with a crazy-paving pattern $(3,9,10)$.

Although common, congestive heart failure and cardiogenic pulmonary edema have been reported previously as a cause for crazy-paving appearance in only three cases (3). The correlation of congestive heart failure with what was once thought to be an uncommon radiological finding is not very familiar to clinicians and radiologists. This is likely in part attributable to the fact that most patients who have congestive heart failure do not undergo chest CT scanning. Nonetheless, it is important to recognize this association to prevent additional unnecessary tests and to provide prompt and appropriate treatment.

\section{SUMMARY}

The present case illustrates the importance of the correlation of radiographic appearance with clinical observations. It also emphasizes that a crazy-paving pattern on CT scan, once considered to be specific for pulmonary alveolar proteinosis, is now known to be nonspecific and may be seen in association with a wide spectrum of pulmonary diseases.

ACKNOWLEDGEMENTS: Supported by an operating grant from the Canadian Institutes of Health Research Grant to GPD. GPD is a Tier 1 Canada Research Chair in respiration sciences. NM is supported by a fellowship from the SACTA-RASHI Foundation, Israel. 


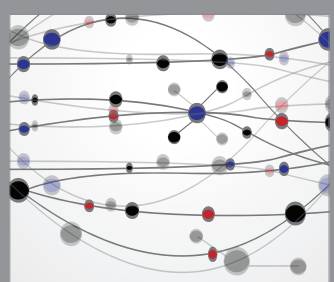

The Scientific World Journal
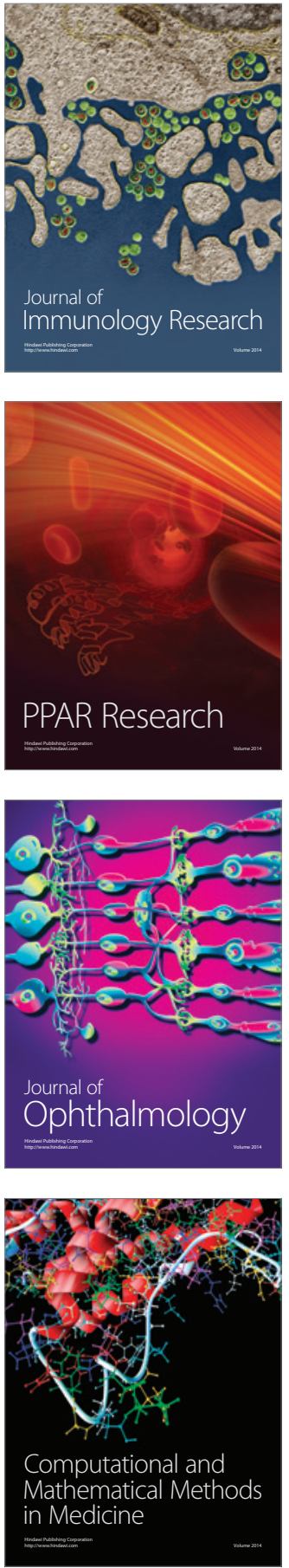

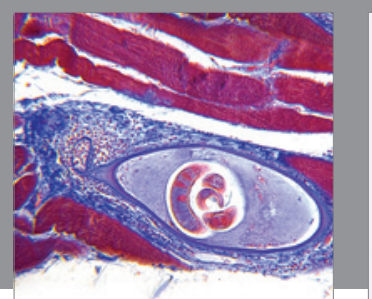

Gastroenterology Research and Practice

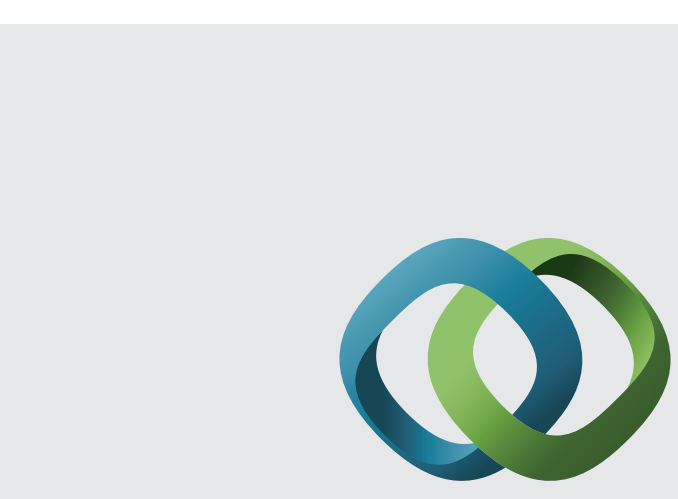

\section{Hindawi}

Submit your manuscripts at

http://www.hindawi.com
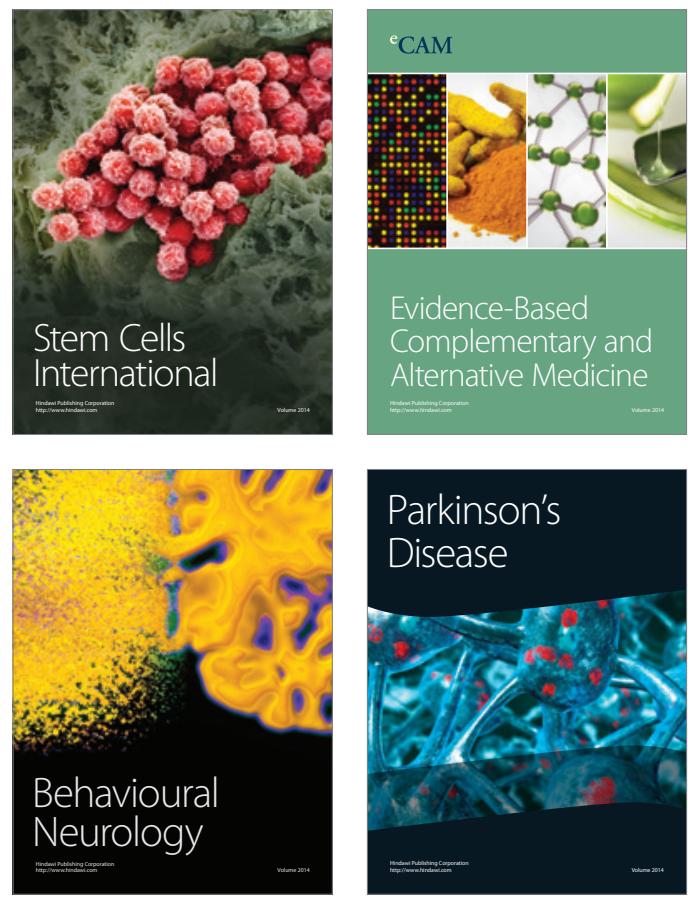
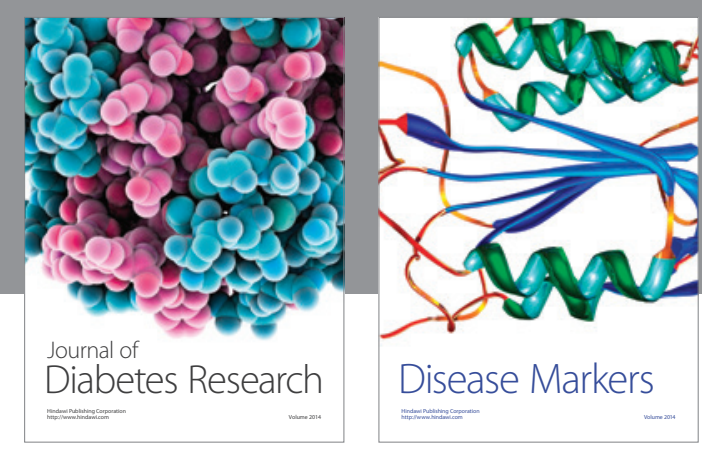

Disease Markers
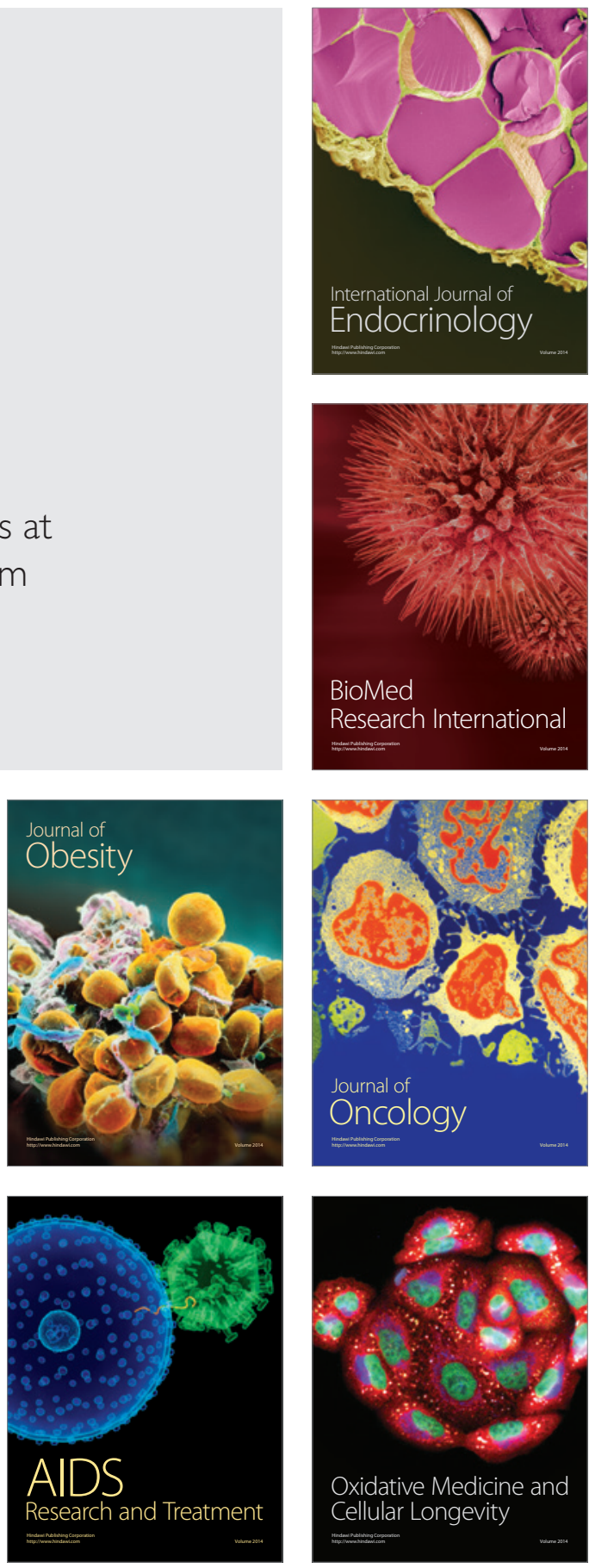